\title{
REVIEW ARTICLE \\ Wild and vaccine-derived poliovirus circulation, and implications for polio eradication
}

\author{
P. L. LOPALCO* \\ Department of Translational Research and New Technologies in Medicine and Surgery University of Pisa, Italy
}

Received 6 June 2016; Final revision 23 September 2016; Accepted 5 October 2016;

first published online 21 November 2016

\section{SUMMARY}

Polio cases due to wild virus are reported by only three countries in the world. Poliovirus type 2 has been globally eradicated and the last detection of poliovirus type 3 dates to November 2012. Poliovirus type 1 remains the only circulating wild strain; between January and September 2016 it caused 26 cases (nine in Afghanistan, 14 in Pakistan, three in Nigeria). The use of oral polio vaccine (OPV) has been the key to success in the eradication effort. However, paradoxically, moving towards global polio eradication, the burden caused by vaccine-derived polioviruses (VDPVs) becomes increasingly important. In this paper circulation of both wild virus and VDPVs is reviewed and implications for the polio eradication endgame are discussed. Between April and May 2016 OPV2 cessation has been implemented globally, in a coordinated switch from trivalent OPV to bivalent OPV. In order to decrease the risk for cVDPV2 re-emergence inactivated polio vaccine (IPV) has been introduced in the routine vaccine schedule of all countries. The likelihood of re-emergence of cVDPVs should markedly decrease with time after OPV cessation, but silent circulation of polioviruses cannot be ruled out even a long time after cessation. For this reason, immunity levels against polioviruses should be kept as high as possible in the population by the use of IPV, and both clinical and environmental surveillance should be maintained at a high level.

Key words: Disease eradication, poliomyelitis, poliovirus, poliovirus vaccines.

\section{Introduction}

Polio eradication has never been so close to being achieved. The last detected case of wild poliovirus (WPV) type 2 dates to 1999 and the Global Commission for the Certification of Poliomyelitis Eradication (GCC), on 20 September 2015, officially concluded that WPV type 2 had been globally eradicated [1]. WPV type 3 was last detected in November 2012 in Nigeria [1]. According to the latest

\footnotetext{
* Address for correspondence: Dr P. L. Lopalco, Via San Zeno 35, 56123 Pisa, Italy.

(Email: pierluigi.lopalco@unipi.it)
}

progress report, in 2015 only 74 paralytic cases due to WPV type 1 were reported from Pakistan and Afghanistan. Since 21 September 2016, 23 additional cases have been reported by those two countries, compared to 41 cases reported during the same period in 2015. In addition, in August 2016, more than 2 years since the last case, three cases of paralytic disease were reported by the health authorities in Nigeria. All the cases reported in 2016 were due to WPV type 1 , the only type currently circulating in the endemic countries, meaning that those countries never stopped indigenous WPV transmission [2].

Trivalent oral polio vaccine (tOPV) use has been the pillar of the successes achieved so far. OPV is 
effective, relatively inexpensive, and does not require either trained health workers or sterile injection equipment to be administered. OPV consists of a mixture of three poliovirus strains attenuated by serial tissue culture passages, and therefore, after oral administration, it mimics natural infection and induces a local immune response in the gut mucosa. Such a feature makes OPV an excellent tool to establish and maintain herd immunity. On the other hand, the capability of OPV viruses to replicate and their transmissiblity becomes a disadvantage during the last phases of the global eradication effort.

OPV viruses are subject to genetic variation through continued mutation and recombination. The attenuating mutation, under selective pressure in the vaccinee's gut, tends to revert towards the wild-type genome, thus potentially resulting in a neurovirulent strain [3]. In fact, mutated vaccine viruses may cause paralytic disease (vaccine-associated paralytic poliomyelitis; VAPP) and may theoretically persist in the environment for a variable period depending on environmental factors [4]. Paradoxically, once the wild strain has been eradicated, OPV viruses would remain the only polioviruses potentially circulating in the world.

As a consequence of the official declaration of WPV type 2 eradication, switching from tOPV to a bivalent formulation not including the type 2 component (bOPV), has occurred globally. Between 17 April and 1 May 2016, 155 countries and territories globally switched from tOPV to bOPV in a synchronized manner. The scope of this paper is to review the current situation on poliovirus circulation, highlighting potential challenges related to vaccination policy changes during the polio eradication endgame.

\section{Vaccine-derived polioviruses (VDPVs)}

As mentioned above, OPV viruses replicate in the gut of vaccinees. In immunocompetent children such replication is limited in time [5] and OPV virus may be excreted for periods up to 30-60 days [6]. During replication cycles, both for stochastic processes and under selection pressure, OPV viruses may progressively mutate, acquire some neurovirulence, and potentially circulate in the community. OPV is responsible for rare cases of VAPP, whose frequency is estimated as 1 case per million births by the WHO [7]. A recent review estimates that such risk is in the range of 2.9$4 \cdot 7$ per million births [8]. VAPP is a rare adverse outcome of polio vaccination occurring in OPV recipients or their close contacts. Risk of VAPP is highest after the first dose and sharply decreases with administration of subsequent doses. There is little evidence of virus circulation after VAPP cases [9]. On the other hand, VDPVs are defined as OPV-derived polioviruses whose sequence divergence from the original OPV strain is such as to indicate prolonged replication or transmission. Since the 'normal' rate of nucleotide substitution for polioviruses amounts to $\sim 1 \%$ per year, poliovirus isolates that differ from the original OPV strain by $>1 \%$ of nucleotide sequence are defined as VDPVs. This threshold has been lowered to $0 \cdot 6 \%$ for type 2 VDPVs (VDPV2) to increase sensitivity for early detection of VDPV2 outbreaks [10]. VDPVs are categorized as 'circulating' (cVDPVs) when there is evidence of community transmission in close contacts. VDPV may be isolated from persons with primary immunodeficiency disorders with prolonged infection after OPV administration. In that case VDPVs are categorized as immunodeficiency-associated VDPVs (iVDPVs). Finally, VDPVs may be classified as 'ambiguous' (aVDPVs) when they are either isolated from persons with no known immunodeficiency or sewage isolates whose source is unknown (sporadic isolation with no genetically linked VDPVs found subsequently) [11].

\section{Public health importance of iVDPVs and cVDPVs}

iVDPVs and cVDPVs share most of their biological characteristics, since both have a variable but significant divergence from the originating OPV strain, and are capable of causing paralytic disease. Genetic differences between iVDPVs and cVDPVs reflect the fact that iVDPVs are the result of prolonged infection in the same individual while cVDPVs have been subjected to the selective pressure during person-to-person transmission. For this reason, vaccine/non-vaccine recombination is very frequently observed in cVDPVs. Recombination of WPVs with other human enteroviruses of species $\mathrm{C}$ (HEV-C), in particular coxsackie A viruses, is frequently the result of person-to-person transmission $[12,13]$. The presence of similar recombination between VDPVs and HEV-C seems to facilitate the emergence of cVDPVs [14]. Although patients infected with iVDPVs may develop paralytic disease and hypothetically present a risk for community spread, cVDPV outbreaks represent the highest public health threat and require the same response as WPVs [7].

\section{cVDPV outbreaks and WPV circulation}

From 12 July 2000 to 12 July 2001, a total of 21 cases of poliomyelitis were confirmed in the Caribbean 


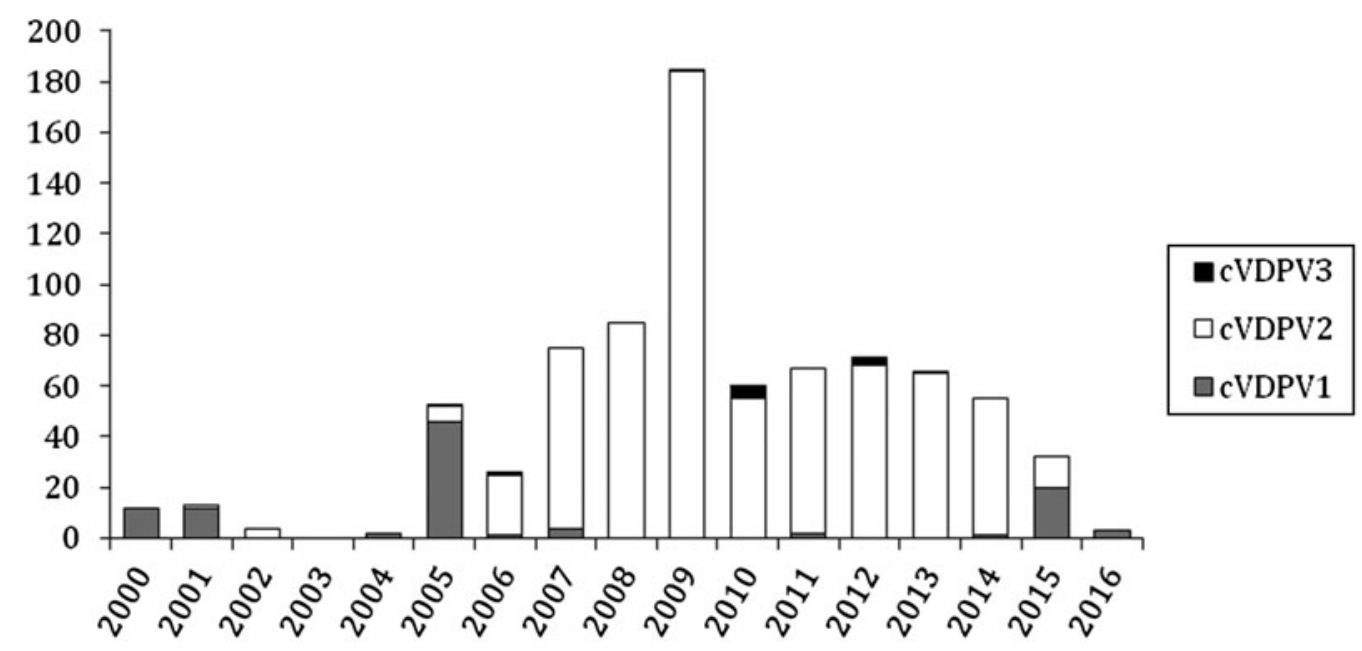

Fig. 1. Number of cVDPV cases globally reported to WHO during the period 2000-2016. (Source: WHO HQ, data as of 21 September 2016.)

island of Hispaniola. This outbreak was associated with an OPV1-derived virus having $1 \cdot 8-4 \cdot 1 \%$ nucleotide substitutions. Such cVDPV1 cases were both clinically and biologically indistinguishable from WP1. The outbreak occurred in an area with low OPV coverage and was the first in the Americas since 1991 [15]. Prior to the Hispaniola outbreak, evidence of circulation of cVDPV2 between 1988 and 1993 was found in Egypt. It was a cVDPV2 having 9397\% nucleotide sequence identity to the Sabin OPV2 strain and was not related to the latest known isolate of WP2 in Egypt that had occurred in 1979. Such evidence was collected ex post and therefore the real extent of the outbreak remains undetermined. Considering several parameters, it is likely that about 12 million children born during the decade 1983-1993 were infected [16]. However, due to improvement in surveillance throughout the 1990s, the absence of any VDPVs in clinical specimens from acute flaccid paralysis (AFP) patients or in environmental samples during the 9 years after 1993 strongly suggests that cVDPV2 spread was stopped [17].

Since the Hispaniola outbreak in 2000-2001, cVDPV outbreaks have been systematically monitored. Figure 1 shows the number of cases globally reported to WHO during the period 2000-2016. Fewer than 90 cases per year are usually reported, with the exception of 2009, when the number of cases peaked, mainly due to a large outbreak in Nigeria [18]. cVDPV2 is the most represented strain, accounting for $85.8 \%$ of all reported cases. Figure 2 shows the geographical distribution of the cVDPV outbreaks over the period. In 2015, circulation of cVDPV2 was documented in Guinea, Myanmar, and Nigeria. The latest cVDPV2 case occurred on 14 December 2015 and was reported from Guinea. In addition, an environmental sample collected in March 2016 in northeastern Nigeria, which recently tested positive for cVDPV2, is an additional evidence of prior circulation that had gone undetected [19]. A total of seven cases were reported in Guinea in 2015, all genetically related to a single case which occurred in August 2014. cVDPV1 outbreaks have been recently reported in Lao People's Democratic Republic (Lao PDR), Madagascar, and Ukraine. The latest cVDPV1 case was reported by Lao PDR on 11 January 2016. Up to 21 September 2016 the total number of cVDPV1 cases in that country amounted to three in 2016 and eight in 2015. No ongoing outbreaks of cVDPV3 have been reported (latest case occurred in 2013) [20].

Ongoing transmission of WPV is currently detected only in Afghanistan, Pakistan, and Nigeria. The total number of WPV1 cases reported in Afghanistan in 2016 is nine. Environmental circulation of WPV1 has also been established in more than one district in 2015 . Fourteen cases of WPV1 were reported in Pakistan in 2016, with the latest onset on 27 July. In Pakistan WPV circulation was never interrupted. According to WHO reports, the Federally Administered Tribal Areas (FATA) is the region with highest virus circulation [19]. After $>2$ years with no wild polio cases, three cases due WP1 have been reported last August in Nigeria. Genetic analysis on the virus suggested that those cases were caused by a virus strain related to the one detected in the northeastern part of the country in 2011, raising 


\begin{tabular}{|c|c|c|c|c|c|c|c|c|c|c|c|c|c|c|c|c|c|c|c|}
\hline CVDPV1 \\
\hline Country & period & $\begin{array}{c}\text { onset of latest } \\
\text { case }\end{array}$ & 2000 & 2001 & 2002 & 2003 & 2004 & 2005 & 2006 & 2007 & 2008 & 2009 & 2010 & 2011 & 2012 & 2013 & 2014 & 2015 & 2016 \\
\hline Laos & $2015 / 16$ & $11-01-16$ & & & & & & & \\
\hline
\end{tabular}

Fig. 2. cVDPV outbreaks, globally reported to WHO, country distribution, 2000-2016. (Source: WHO HQ, data as of 21 September 2016.)

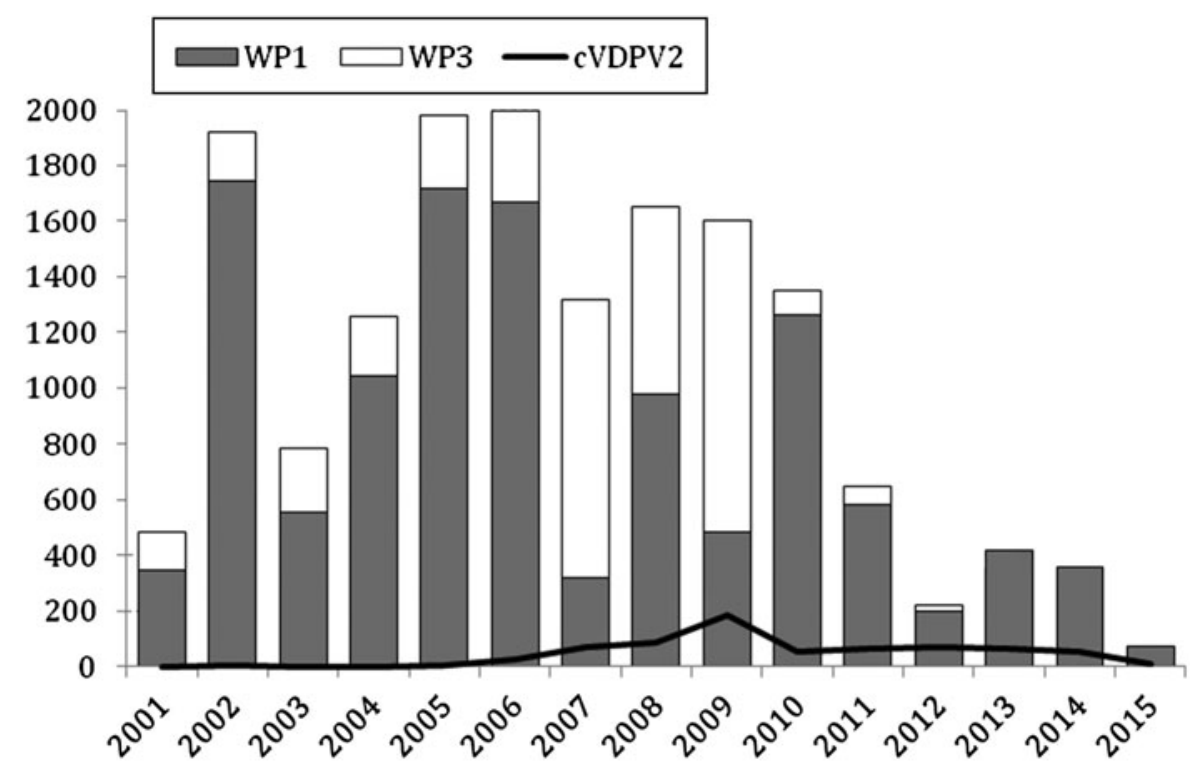

Fig. 3. Number of cases of wild poliovirus types 1 and 3 and cVDPV2 globally reported to WHO during the period 2001-2015. (Source: WHO HQ, data as of 21 September 2016.)

the issue of silent circulation of the virus in that area for a lengthy period of time. Figure 3 shows the number of cases of WPV types 1 and 3 globally reported to the WHO during the period 2001-2015, compared to
cVDPV2 cases (the only type 2 poliovirus currently circulating). Starting in 2009, a strong decrease of WP3 cases has been observed globally, with the last case reported in 2012. Considering these three polio strains, since 2013 


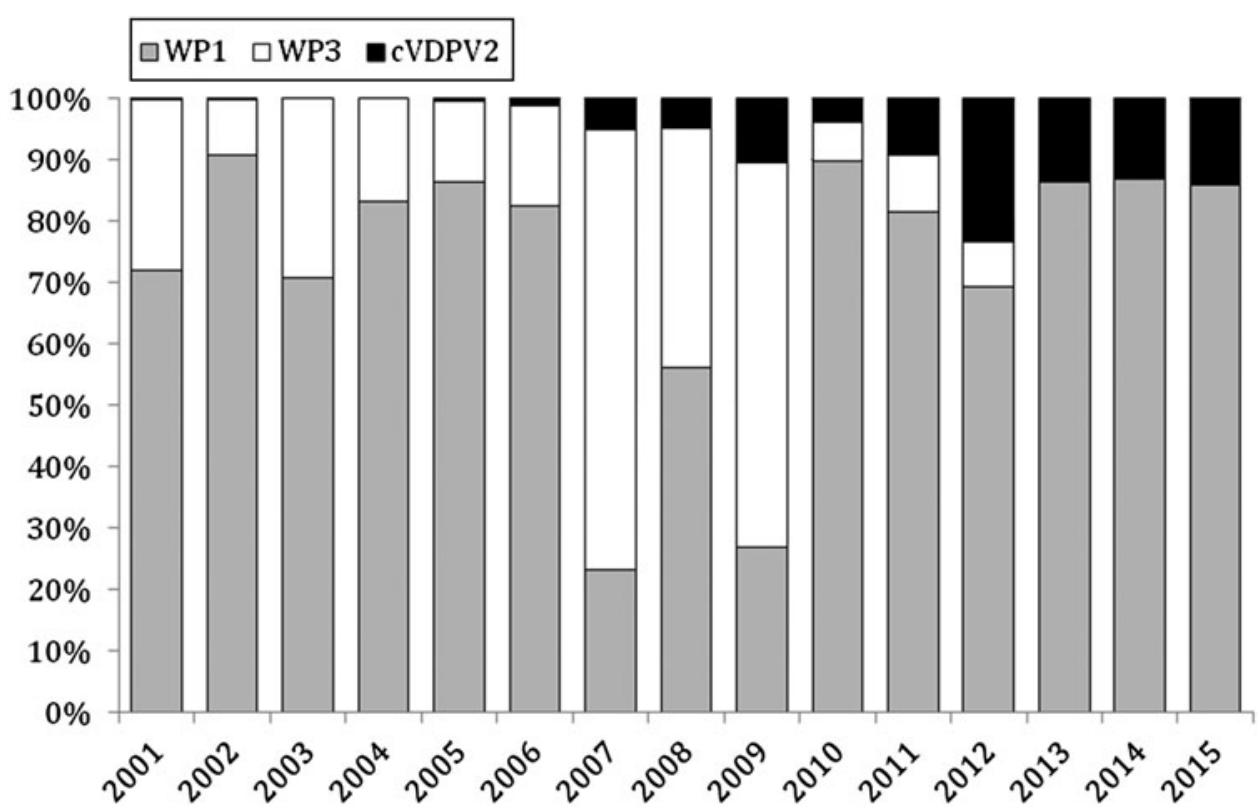

Fig. 4. Proportion of cases of wild poliovirus types 1 and 3 and cVDPV2 globally reported to WHO during the period 2001-2015. (Source: WHO HQ, data as of 21 September 2016.)

WP1 and cVDPV2 are the only polioviruses causing clinical cases. During this period, cVDPV2 accounted for about $13 \%$ of the globally reported cases (Fig. 4).

\section{Public health importance of cVDPV2 after OPV2 cessation}

The possibility of eradicating WPV by the end of 2016 is becoming a reality. Moving towards WPV eradication, the relative importance of cVDPV tends to increase. In particular, after declaration of WPV2 eradication and subsequent OPV2 cessation, there will be some challenges in the future.

Even if the quality of surveillance in the countries with recently documented cVDPV2 circulation is good, detection of either new cases or environmental presence of cVDPV2 cannot be ruled out, even in the absence of new OPV2 administration. Persistence of cVDPV2 after OPV2 cessation will depend on both person-to-person transmission in the underimmunized population groups, and environmental persistence. Persistence of polioviruses in the environment is limited in time - from about 1 week to 1 month - being rapidly inactivated, especially under tropical conditions $[4,21]$. Therefore, the main determinants for continuous circulation of cVDPV2 are linked to population characteristics such as population clustering or population turnover [22], but primarily to the proportion of susceptible individuals.
Following cessation, OPV2 needs to be replaced by inactivated polio vaccine (IPV) use. According to WHO guidelines on OPV2 cessation, countries switching from tOPV to bOPV, should have already introduced at least one IPV dose into the routine immunization schedule in order to keep high the immunity against type 2 viruses immediately after OPV2 cessation. On the other hand, effectiveness of IPV in preventing gut replication and the shedding of polioviruses is controversial. Mathematical modelling shows that use of one-dose IPV after OPV cessation in areas at higher risk of poliovirus circulation has a modest effect on the risk of cVDPV outbreaks [23]. In 2013, documented WP1 circulation in the environment in a community of fully IPV-vaccinated children in Israel [24] was interrupted only after vaccinating about 1 million children aged $<10$ years with bOPV [25]. Therefore, even in the presence of relatively high population immunity, use of IPV alone failed to prevent silent circulation of WP1. By contrast, recent experimental evidence collected in Latin America shows some degree of intestinal immunity after monovalent OPV2 (mOPV2) trials in young children vaccinated with a sequential bOPV-two IPV schedule [26]. In developed countries using a full IPV schedule over a lengthy period, neurovirulent polioviruses may be isolated from the environment, but the immunity levels in the population, together with high hygiene levels, prevent the occurrence of continuous circulation and 
clinical cases [27, 28]. Similarly, during the polio outbreak that occurred in The Netherlands in 1992, disease was confined to unvaccinated communities and did not spread in the rest of the country where a full IPV schedule had been adopted [29]. Therefore, if WPVs are introduced into communities with good sanitation and hygiene, high levels of coverage with IPV, in addition to preventing polio cases, may potentially stop poliovirus spread. It is very likely that wide use of IPV after OPV2 cessation will be able to control some residual circulation of cVDPV2 in the population.

\section{Conclusions}

Assessing the risk of cVDPV2 re-emergence and further circulation after OPV2 cessation is not an easy task, and any attempt cannot be other than speculative. Due to the interconnections and rapid movement of people, long-lasting presence of cVDPV2 in a specific network of people cannot be ruled out. Silent virus circulation may occur and can be detected only by the means of high-quality environmental surveillance [24]. In fact, AFP surveillance alone is not sufficient to detect early circulation of polioviruses and to monitor the extent of environmental circulation. Moreover, modelling suggests that silent circulation of polioviruses may be ruled out, with an error of $5 \%$, only after a case-free period of 3 years [30]. On the other hand, in the presence of good levels of population immunity, the risk of paralytic cases is believed to be extremely low. In any case, contingency plans based on stockpiling and deployment of mOPV2 are necessary, since the response to a single cVDPV2 case should be the same as to a WPV outbreak. It should be noted that, according to the eradication programme, one single polio case must be considered an outbreak.

Implementation of International Health Regulations demonstrated an excellent efficacy in supporting the polio endgame. In fact, the declaration that the international spread of polio constituted a Public Health Emergency of International Concern (PHEIC), and the subsequent recommendation by the WHO in 2014, determined strong progress towards the interruption of WPV transmission [31]. Similarly, countries detecting cVDPV cases should react by following the same WHO temporary recommendations, declaring the outbreak a national public health emergency and considering vaccination of all international travellers [32].

Implemented measures and recommendations should be stringent enough to prevent extensive spread of cVDPVs until polio vaccination ends. Several years after global cessation of vaccination, when several fully susceptible birth cohorts will have accumulated, a completely different situation might be encountered. In that case the danger would be due to the accidental release or long-term excretion of potential polioviruses by immunocompromised individuals [33]. Accidental release cannot be completely ruled out even in the best virus-containment scenario. Recently, accidental release of WPV3 in the environment occurred in Belgium, close to the Dutch border. This has not resulted in detectable levels of poliovirus in any of the samples taken after the incident and no signs of poliovirus circulation were found in the Dutch 'Bible belt', well known as home to large underimmunized communities [34]. In addition, inadvertent tOPV use should be prevented especially in areas at higher risk of cVDPV circulation by the means of tOPV withdrawal from all OPV stocks [35].

In conclusion, the likelihood of re-emergence of cVDPVs should markedly decrease with time after OPV cessation. Immunity levels against polioviruses should be kept as high as possible in the population through the continuous use of IPV, which may represent a challenge in those countries where high coverage levels with IPV cannot be sustained. Effective contingency plans, supported by both clinical and environmental surveillance, should be kept in place.

\section{Acknowledgements}

We thank Dr Maria Giulia Bianchi, Dr Silvia Gandolfo, and Dr Filippo Giardi for assistance with polio cases review, and Professor Gaetano Privitera for comments that greatly improved the manuscript.

\section{Declaration of Interest}

None.

\section{References}

1. Polio Global Eradication Initiative. Global eradication of wild poliovirus type 2 declared (http://www.polioeradication.org/mediaroom/newsstories/Global-eradicationofwild-poliovirus-type-2-declared/tabid/526/news/1289/ Default.aspx\#sthash.TWdccMBL.dpuf). Accessed 31 May 2016.

2. Polio Global Eradication Initiative. Wild poliovirus type 1 and circulating vaccine-derived poliovirus cases (http://www.polioeradication.org/Dataandmonitoring/ Poliothisweek.aspx\#sthash.1c5 0b7hA.dpuf). Accessed 21 September 2016. 
3. Minor PD. The molecular biology of polio vaccines. Journal of General Virology 1992; 73: 3065-3077.

4. Fine PEM, Carneiro IAM. Transmissibility and persistence of oral polio vaccine viruses: implications for the global poliomyelitis eradication initiative. American Journal of Epidemiology 1999; 150: 1001-1021.

5. Martin J. Vaccine-derived poliovirus from long term excretors and the endgame of polio eradication. Biologicals 2006; 34: 117-122.

6. Abraham R, et al. Shedding of virulent poliovirus revertants during immunization with oral poliovirus vaccine after prior immunization with inactivated polio vaccine. Journal of Infectious Diseases 1993; 168: 1105-1109.

7. Minor P. Vaccine-derived poliovirus (VDPV): impact on poliomyelitis eradication. Vaccine 2009; 27: 26492652.

8. Platt LR, Estívariz CF, Sutter RW. Vaccine-associated paralytic poliomyelitis: a review of the epidemiology and estimation of the global burden. Journal of Infectious Diseases 2014; 210 (Suppl. 1): S380-389.

9. Korotkova EA, et al. A cluster of paralytic poliomyelitis cases due to transmission of slightly diverged Sabin-2 vaccine virus. Journal of Virology 2016; 90: 5978-5988.

10. Wassilak S, et al. Outbreak of type 2 vaccine-derived poliovirus in Nigeria: Emergence and widespread circulation in an underimmunized population. Journal of Infectious Diseases 2011; 203: 898-909.

11. CDC. Update on vaccine-derived polioviruses worldwide, January 2008-June 2009. Morbidity and Mortality Weekly Report 2009; 58: 1002-1006.

12. Liu H-M, et al. Serial recombination during circulation of type 1 wild-vaccine recombinant polioviruses in China. Journal of Virology 2003; 77: 10994-11005.

13. Combelas $\mathbf{N}$, et al. Recombination between poliovirus and coxsackie a viruses of species $\mathrm{C}$ : a model of viral genetic plasticity and emergence. Viruses 2011; 3: 1460-1484.

14. Burns CC, et al. Vaccine-derived polioviruses. Journal of Infectious Diseases 2014; 210 (Suppl. 1): S283-S293.

15. CDC. Public Health Dispatch: Update: outbreak of poliomyelitis - Dominican Republic and Haiti, 20002001. Morbidity and Mortality Weekly Report 2001; 50: $855-856$.

16. Wringe A, et al. Estimating the extent of vaccine-derived poliovirus infection. PLoS ONE 2008; 3: e3433.

17. Yang CF, et al. Circulation of endemic type 2 vaccinederived poliovirus in Egypt from 1983 to 1993. Journal of Virology 2003; 77: 8366-8377.

18. Polio Global Eradication Initiative. Circulating vaccinederived polioviruses. (http://www.polioeradication.org/ Dataandmonitoring/Poliothisweek/Circulatingvaccine derivedpoliovirus.aspx). Accessed 30 May 2016.

19. Morales M, Tangermann RH, Wassilak SG. Progress toward polio eradication-worldwide, 2015-2016. Morbidity and Mortality Weekly Report 2016; 65: 470-473.

20. Polio Global Eradication Initiative. Polio public health emergency (http://www.polioeradication.org/Keycountries/ PolioEmergency.aspx). Accessed 30 May 2016.
21. Yates MV, Gerba CP, Kelley LM. Virus persistence in groundwater. Applied and Environmental Microbiology 1985; 49: 778-781.

22. Yorke JA, et al. Seasonality and the requirements for perpetuation and eradication of viruses in populations. American Journal of Epidemiology 1979; 109: 103-23.

23. Duintjer Tebbens RJ, Thompson KM. Modeling the potential role of inactivated poliovirus vaccine to manage the risks of oral poliovirus vaccine cessation. Journal of Infectious Diseases 2014; 210 (Suppl. 1): S485-S497.

24. Anis E, et al. Insidious reintroduction of wild poliovirus into Israel, 2013. Eurosurveillance 2013; 18: pii $=20586$.

25. Kaliner E, et al. The Israeli public health response to wild poliovirus importation. Lancet Infectious Diseases 2015; 15: 1236-1242.

26. Asturias EJ, et al. Humoral and intestinal immunity induced by new schedules of bivalent oral poliovirus vaccine and one or two doses of inactivated poliovirus vaccine in Latin American infants: an openlabel randomised controlled trial. Lancet 2016; 388: 158-169.

27. Al-Hello H, et al. Highly divergent type 2 and 3 vaccinederived polioviruses isolated from sewage in Tallinn, Estonia. Journal of Virology 2013; 87: 13076-13080.

28. Roivainen M, et al. Highly divergent neurovirulent vaccine-derived polioviruses of all three serotypes are recurrently detected in Finnish sewage. Eurosurveillance 2010; 15: pii $=19566$.

29. Conyn-van Spaendonck MA, et al. Circulation of poliovirus during the poliomyelitis outbreak in The Netherlands in 1992-1993. American Journal of Epidemiology 1996; 143: 929-935.

30. Eichner M, Dietz K. Eradication of poliomyelitis: when can one be sure that polio virus transmission has been terminated? American Journal of Epidemiology 1996; 143: $816-822$.

31. WHO. WHO statement on the meeting of the International Health Regulations Emergency Committee concerning the international spread of wild poliovirus (http://www.who.int/mediacentre/news/statements/2014/ polio-20140505/en/). Accessed 30 May 2016.

32. WHO. Statement on the 10th IHR Emergency Committee regarding the international spread of poliovirus (http://www.who.int/mediacentre/news/statements/ 2016/10th-ihr-emergency/en/). Accessed 20 September 2106.

33. Shaghaghi M, et al. Vaccine-derived polioviruses and children with primary immunodeficiency, Iran, 19952014. Emerging Infectious Diseases 2016; 22: 1712-1719.

34. Duizer E, et al. Risk assessment, risk management and risk-based monitoring following a reported accidental release of poliovirus in Belgium, September to November 2014. Eurosurveillance 2016; 21: pii $=30169$.

35. Duintjer Tebbens RJ, Hampton LM, Thompson KM. Implementation of coordinated global serotype 2 oral poliovirus vaccine cessation: risks of inadvertent trivalent oral poliovirus vaccine use. BMC Infectious Diseases 2016; 16: 237. 